\title{
Structural sequences for primes using right-end-digits
}

\author{
J. V. Leyendekkers ${ }^{1}$ and A. G. Shannon ${ }^{2}$ \\ ${ }^{1}$ Faculty of Science, The University of Sydney, NSW 2006, Australia \\ e-mail: jeanvaldek@gmail.com \\ ${ }^{2}$ Warrane College, The University of New South Wales, NSW 2033, Australia \\ e-mail: tshannon38@gmail.com
}

Received: 4 January 2017

Accepted: 29 October 2017

\begin{abstract}
Integers are expressed in the form $n R$ where $R$ represents the right-end-digits and $n$ represents the digits to the left of $R . n$ can be classified by the sequences $\{3 t\},\{3 t+1\}$, $\{3 t+2\}$. When $n=3 t+2$, no primes with $R=1$ or 7 can be formed with these $n$; when $n=3 t$ no primes can be formed with $R=3$ or 9 , but when $n=3 t+1$, all REDs can form a prime within the constraints of imbedded sequences.
\end{abstract}

Keywords: Prime numbers, Composite numbers, Right-end-digits, Integer structure.

2010 Mathematics Subject Classification: 11B50.

\section{Introduction}

Integers are composed of a number of digits with a structure where the right-end-digits (REDs) are independent of the size of the integer, so that it is convenient to consider their form as $n R$ where $R$ is the RED and $n$ contains the remaining digits to the left of $R$. For example, the integer 279856321 has $R=1$ and $n=27985632$.

The $n$ part belongs to the sequences $\{3 t\},\{3 t+1\},\{3 t+2\}$ and imbedded sequences of the form $\{a+b j\}$ where $a<b$ and $3 \mathrm{lb}$, with $a$ and $b$ constant for a given sequences, $j$ a variable. 


\section{Imbedded sequences which block prime formation for particular REDs}

These concepts are best illustrated by considering the REDs 1 and 7, and 3 and 9, as separate pairs since when $n \in\{3 t\}$ with REDs of 3 or 9 , these integers can never be prime, while REDs 1 and 7 with $n \in\{3 t+2\}$ can never form prime integers, but when $n \in\{3 t+1\}$, the integers could be primes. This can be more finely tuned with imbedded sequences which demonstrate selectivity for certain REDs, namely when $n$ conforms to certain $(a, b)$ pairs, the REDs will always be composite (Table 1).

To illustrate this structure $n$ values for the two pairs of REDs 1,7 and 3,9 are listed in Tables 2 (a) and (b) and Table 3 for $n=1$ to 100 with the imbedded sequences which prevent the formation of primes. Larger primes composed of 30 and 50 digits are shown in Tables 4 and 5.

\begin{tabular}{|c|c|l|}
\hline \multirow{2}{*}{ RED } & $\begin{array}{c}\text { Main } \\
\text { sequence }\end{array}$ & \multicolumn{1}{|c|}{$(\boldsymbol{a}, \boldsymbol{b})$} \\
\hline \multirow{2}{*}{1} & $3 t$ & $(3,39),(3,93)[n>3],(9,21),(9,39),(12,33),(36,57),(39,51),(84,87)$ \\
\cline { 2 - 4 } & $3 t+1$ & $(1,66)[n>1],(16,21),(16,69),(22,39),(22,51),(34,66)$ \\
\hline \multirow{2}{*}{7} & $3 t$ & $(0,21),(18,33),(18,51),(24,39),(24,57),(66,69)$ \\
\cline { 2 - 4 } & $3 t+1$ & $(1,51)[n>1],(7,21),(7,66),(37,39),(43,57)$ \\
\hline \multirow{2}{*}{1,7} & $3 t+2$ & No primes formed \\
\hline \multirow{2}{*}{3} & $3 t+1$ & $(1,39),(13,21),(13,57),(25,33),(25,69),(49,51)$ \\
\cline { 2 - 4 } & $3 t+2$ & $(2,69)[n>2),(14,33,(14,39),(20,21),(32,51),(32,57)$ \\
\hline \multirow{2}{*}{9} & $3 t+1$ & $\begin{array}{l}(1,57)[n>1],(4,21),(16,39),(28,51),(31,33),(52,69),(64,231), \\
(79,102)\end{array}$ \\
\cline { 2 - 4 } & $3 t+2$ & $(11,21),(11,51),(20,33),(20,57),(29,39),(29,69),(32,42),(89,93)$ \\
\hline 3,9 & $3 t$ & No primes formed \\
\hline
\end{tabular}

Table 1. Imbedded sequences blocking prime formation for particular REDs 


\begin{tabular}{|c|c|c|c|c|c|c|c|c|c|c|c|c|c|c|}
\hline \multirow{2}{*}{$n$} & \multirow{2}{*}{$\boldsymbol{R}$} & \multirow{2}{*}{ Type } & \multicolumn{2}{|c|}{ Sequence } & \multirow{2}{*}{$n$} & \multirow{2}{*}{$\boldsymbol{R}$} & \multirow{2}{*}{ Type } & \multicolumn{2}{|c|}{ Sequence } & \multirow{2}{*}{$n$} & \multirow{2}{*}{$\boldsymbol{R}$} & \multirow{2}{*}{ Type } & \multicolumn{2}{|c|}{ Sequence } \\
\hline & & & $n=$ & $R \neq$ & & & & $n=$ & $R \neq$ & & & & $n=$ & $R \neq$ \\
\hline \multirow[t]{2}{*}{3} & 1 & $p$ & & & \multirow[t]{2}{*}{36} & 1 & $c$ & $36+57 j$ & 1 & \multirow[t]{2}{*}{72} & 1 & $c$ & $9+21 j$ & 1 \\
\hline & 7 & $p$ & & & & 7 & $p$ & & & & 7 & $p$ & & \\
\hline \multirow[t]{2}{*}{6} & 1 & $p$ & & & \multirow[t]{2}{*}{39} & 1 & $c$ & $39+51 j$ & 1 & \multirow[t]{2}{*}{75} & 1 & $p$ & & \\
\hline & 7 & $p$ & & & & 7 & $p$ & & & & 7 & $p$ & & \\
\hline \multirow[t]{2}{*}{9} & 1 & $c$ & $9+21 j$ & 1 & \multirow[t]{2}{*}{42} & 1 & $p$ & & & \multirow[t]{2}{*}{78} & 1 & $c$ & $12+33 j$ & 1 \\
\hline & 7 & $p$ & & & & 7 & $c$ & $21 j$ & 7 & & 7 & $p$ & & \\
\hline \multirow[t]{2}{*}{12} & 1 & $c$ & $12+33 j$ & 1 & \multirow[t]{2}{*}{45} & 1 & $c$ & $12+33 j$ & 1 & \multirow[t]{2}{*}{81} & 1 & $p$ & & \\
\hline & 7 & $p$ & & & & 7 & $p$ & & & & 7 & $c$ & $24+57 j$ & 7 \\
\hline \multirow[t]{2}{*}{15} & 1 & $p$ & & & \multirow[t]{2}{*}{48} & 1 & $c$ & $9+39 j$ & 1 & \multirow[t]{2}{*}{84} & 1 & $c$ & $84+87 j$ & 1 \\
\hline & 7 & $p$ & & & & 7 & $p$ & & & & 7 & $c$ & $18+33 j$ & 7 \\
\hline \multirow[t]{2}{*}{18} & 1 & $p$ & & & \multirow[t]{2}{*}{51} & 1 & $c$ & $9+21 j$ & 1 & 87 & 1 & $c$ & $9+39 j$ & 1 \\
\hline & 7 & $c$ & $18+33 j$ & 7 & & 7 & $c$ & $18+33 j$ & 7 & & 7 & $p$ & & \\
\hline 21 & 1 & $p$ & & & 54 & 1 & $p$ & & & 90 & 1 & $c$ & $39+51 j$ & 1 \\
\hline & 7 & $c$ & $21 j$ & 7 & & 7 & $p$ & & & & 7 & $p$ & & \\
\hline 24 & 1 & $p$ & & & 57 & 1 & $p$ & & & 93 & 1 & $c$ & $\begin{array}{c}36+57 j \\
9+21 j\end{array}$ & 1 \\
\hline & 7 & $c$ & $24+57 j$ & 7 & & 7 & $p$ & & & & 7 & $p$ & & \\
\hline 27 & 1 & $p$ & & & 60 & 1 & $p$ & & & 96 & 1 & $c$ & $3+93 j$ & 1 \\
\hline & 7 & $p$ & & & & 7 & $p$ & & & & 7 & $p$ & & \\
\hline 30 & 1 & $c$ & $9+21 j$ & 1 & 63 & 1 & $p$ & & & 99 & 1 & $p$ & & \\
\hline & 7 & $p$ & & & & 7 & $c$ & $21 j$ & 7 & & 7 & $p$ & & \\
\hline 33 & 1 & $p$ & & & 66 & 1 & $p$ & & & 102 & 1 & $p$ & & \\
\hline & 7 & $p$ & & & & 7 & $c$ & $66+69 j$ & 7 & & 7 & $c$ & $24+39 j$ & 7 \\
\hline & & & & & 69 & 1 & $p$ & & & & & & & \\
\hline & & & & & & 7 & $c$ & $18+51 j$ & 7 & & & & Usill & \\
\hline
\end{tabular}

Table 2 (a). $n=3 t, R \neq 1,7$ means that no primes are formed with these $R$ values and the $n$ sequence $(a+b j)$ 
On the other hand Table $2 \mathrm{~b}: R \neq 1,7$ indicates the imbedded sequences which prevent the $n$ from ever forming primes with these $n$ from $n=1$ to $n=100$ in steps of 3 .

\begin{tabular}{|c|c|c|c|c|c|c|c|c|c|c|c|c|c|c|}
\hline \multirow{2}{*}{$n$} & \multirow{2}{*}{$\boldsymbol{R}$} & \multirow{2}{*}{ Type } & \multicolumn{2}{|c|}{ Sequence } & \multirow{2}{*}{$n$} & \multirow{2}{*}{$R$} & \multirow{2}{*}{ Type } & \multicolumn{2}{|c|}{ Sequence } & \multirow{2}{*}{$n$} & \multirow{2}{*}{$R$} & \multirow{2}{*}{ Type } & \multicolumn{2}{|c|}{ Sequence } \\
\hline & & & $n=$ & $R \neq$ & & & & $n=$ & $R \neq$ & & & & $N=$ & $R \neq$ \\
\hline \multirow[t]{2}{*}{1} & 1 & $p$ & & & \multirow[t]{2}{*}{34} & 1 & $c$ & $34+66 j$ & 1 & \multirow[t]{2}{*}{70} & 1 & $p$ & & \\
\hline & 7 & $p$ & & & & 7 & $p$ & & & & 7 & $c$ & $7+21 j$ & 7 \\
\hline \multirow[t]{2}{*}{4} & 1 & $p$ & & & \multirow[t]{2}{*}{37} & 1 & $c$ & $16+21 j$ & 1 & \multirow[t]{2}{*}{73} & 1 & $c$ & $22+51 j$ & 1 \\
\hline & 7 & $p$ & & & & 7 & $c$ & $37+39 j$ & 7 & & 7 & $c$ & $7+66 j$ & 7 \\
\hline \multirow[t]{2}{*}{7} & 1 & $p$ & & & \multirow[t]{2}{*}{40} & 1 & $p$ & & & \multirow[t]{2}{*}{76} & 1 & $p$ & & \\
\hline & 7 & $c$ & $7+21 j$ & 7 & & 7 & $c$ & $7+33 j$ & 7 & & 7 & $c$ & $37+39 j$ & 7 \\
\hline \multirow[t]{2}{*}{10} & 1 & $p$ & & & \multirow[t]{2}{*}{43} & 1 & $p$ & & & \multirow[t]{2}{*}{79} & 1 & $c$ & $16+21 j$ & 1 \\
\hline & 7 & $p$ & & & & 7 & $c$ & $43+57 j$ & 7 & & 7 & $p$ & & \\
\hline \multirow[t]{2}{*}{13} & 1 & $p$ & & & \multirow[t]{2}{*}{46} & 1 & $p$ & & & \multirow[t]{2}{*}{82} & 1 & $p$ & & \\
\hline & 7 & $p$ & & & & 7 & $p$ & & & & 7 & $p$ & & \\
\hline \multirow[t]{2}{*}{16} & 1 & $c$ & $16+21 j$ & 1 & \multirow[t]{2}{*}{49} & 1 & $p$ & & & 85 & 1 & $c$ & $16+69 j$ & 1 \\
\hline & 7 & $p$ & & & & 7 & $c$ & $7+21 j$ & 7 & & 7 & $p$ & & \\
\hline 19 & 1 & $p$ & & & 52 & 1 & $p$ & & & 88 & 1 & $p$ & & \\
\hline & 7 & $p$ & & & & 7 & $c$ & $1+51 j$ & 7 & & 7 & $p$ & & \\
\hline 22 & 1 & $c$ & $22+39 j$ & 1 & 55 & 1 & $c$ & $55+57 j$ & 1 & 91 & 1 & $p$ & & \\
\hline & 7 & $p$ & & & & 7 & $p$ & & & & 7 & $c$ & $7+21 j$ & 7 \\
\hline 25 & 1 & $p$ & & & 58 & 1 & $c$ & $16+21 j$ & 1 & 94 & 1 & $p$ & & \\
\hline & 7 & $p$ & & & & 7 & $p$ & & & & 7 & $p$ & & \\
\hline 28 & 1 & $p$ & & & 61 & 1 & $c$ & $22+39 j$ & 1 & 97 & 1 & $p$ & & \\
\hline & 7 & $c$ & $7+21 j$ & 7 & & 7 & $p$ & & & & 7 & $p$ & & \\
\hline 31 & 1 & $p$ & & & 64 & 1 & $p$ & & & 100 & 1 & $c$ & $16+21 j$ & 1 \\
\hline & 7 & $p$ & & & & 7 & $p$ & & & & 7 & $c$ & $43+57 j$ & 7 \\
\hline & & & & & 67 & 1 & $c$ & $1+66 j$ & 1 & & & & & \\
\hline & & & & & & 7 & $p$ & & & & & & posite & \\
\hline
\end{tabular}

Table 2 (b). $n=3 t+1, R \neq 1,7$ indicates that the imbedded sequences prevent the $n$ from ever forming primes with these $n$ 


\begin{tabular}{|c|c|c|c|c|c|c|c|c|c|c|c|c|c|c|}
\hline \multirow{2}{*}{$n$} & \multirow{2}{*}{$R$} & \multirow{2}{*}{ Type } & \multicolumn{2}{|c|}{ Sequence } & \multirow{2}{*}{$n$} & \multirow{2}{*}{$R$} & \multirow{2}{*}{ Type } & \multicolumn{2}{|c|}{ Sequence } & \multirow{2}{*}{$n$} & \multirow{2}{*}{$\boldsymbol{R}$} & \multirow{2}{*}{ Type } & \multicolumn{2}{|c|}{ Sequence } \\
\hline & & & $n=$ & $R \neq$ & & & & $n=$ & $R \neq$ & & & & $n=$ & $R \neq$ \\
\hline \multirow[t]{2}{*}{1} & 3 & $p$ & & & \multirow[t]{2}{*}{34} & 3 & $c$ & $13+21 j$ & 3 & \multirow[t]{2}{*}{70} & 3 & $c$ & $13+57 j$ & 3 \\
\hline & 9 & $p$ & & & & 9 & $p$ & & & & 9 & $p$ & & \\
\hline \multirow[t]{2}{*}{4} & 3 & $p$ & & & \multirow[t]{2}{*}{37} & 3 & $p$ & & & \multirow[t]{2}{*}{73} & 3 & $p$ & & \\
\hline & 9 & $c$ & $4+21 j$ & 9 & & 9 & $p$ & & & & 9 & $p$ & & \\
\hline \multirow[t]{2}{*}{7} & 3 & $p$ & & & \multirow[t]{2}{*}{40} & 3 & $c$ & $1+39 j$ & 3 & \multirow[t]{2}{*}{76} & 3 & $c$ & $13+21 j$ & 3 \\
\hline & 9 & $p$ & & & & 9 & $p$ & & & & 9 & $p$ & & \\
\hline \multirow[t]{2}{*}{10} & 3 & $p$ & & & \multirow[t]{2}{*}{43} & 3 & $p$ & & & \multirow[t]{2}{*}{79} & 3 & $c$ & $1+39 j$ & 3 \\
\hline & 9 & $p$ & & & & 9 & $p$ & & & & 9 & $c$ & $79+102 j$ & 9 \\
\hline \multirow[t]{2}{*}{13} & 3 & $c$ & $13+21 j$ & 3 & \multirow[t]{2}{*}{46} & 3 & $p$ & & & \multirow[t]{2}{*}{82} & 3 & $p$ & & \\
\hline & 9 & $p$ & & & & 9 & $c$ & $4+21 j$ & 9 & & 9 & $p$ & & \\
\hline \multirow[t]{2}{*}{16} & 3 & $p$ & & & \multirow[t]{2}{*}{49} & 3 & $c$ & $49+51 j$ & 3 & 85 & 3 & $p$ & & \\
\hline & 9 & $c$ & $16+39 j$ & 9 & & 9 & $p$ & & & & 9 & $p$ & & \\
\hline 19 & 3 & $p$ & & & 52 & 3 & $p$ & & & 88 & 3 & $p$ & & \\
\hline & 9 & $p$ & & & & 9 & $c$ & $52+69 j$ & 9 & & 9 & $c$ & $4+21 j$ & 9 \\
\hline 22 & 3 & $p$ & & & 55 & 3 & $c$ & $13+21 j$ & 3 & 91 & 3 & $c$ & $25+33 j$ & 3 \\
\hline & 9 & $p$ & & & & 9 & $c$ & $16+39 j$ & 9 & & 9 & $p$ & & \\
\hline 25 & 3 & $c$ & $5+33 j$ & 3 & 58 & 3 & $c$ & $25+33 j$ & 3 & 94 & 3 & $c$ & $25+69 j$ & 3 \\
\hline & 9 & $c$ & $4+21 j$ & 9 & & 9 & $c$ & $1+57 j$ & 9 & & 9 & $c$ & $16+39 j$ & 9 \\
\hline 28 & 3 & $p$ & & & 61 & 3 & $p$ & & & 97 & 3 & $c$ & $13+21 j$ & 3 \\
\hline & 9 & $c$ & $28+51 j$ & 9 & & 9 & $p$ & & & & 9 & $c$ & $31+33 j$ & 9 \\
\hline 31 & 3 & $p$ & & & 64 & 3 & $p$ & & & 100 & 3 & $c$ & $49+51 j$ & 3 \\
\hline & 9 & $c$ & $31+33 j$ & 9 & & 9 & $c$ & $31+33 j$ & 9 & & 9 & $p$ & & \\
\hline & & & & & 67 & 3 & $p$ & & & & & & & \\
\hline & & $p: 1$ & ime & & & 9 & $c$ & $4+21 j$ & 9 & & & $c: \operatorname{com}$ & osite & \\
\hline
\end{tabular}

Table 3 (a). $n=3 t+1$ 


\begin{tabular}{|c|c|c|c|c|c|c|c|c|c|c|c|c|c|c|}
\hline \multirow[b]{2}{*}{$n$} & \multirow[b]{2}{*}{$\boldsymbol{R}$} & \multirow[b]{2}{*}{ Type } & \multicolumn{2}{|c|}{ Sequence } & \multirow[b]{2}{*}{$n$} & \multirow[b]{2}{*}{$R$} & \multirow[b]{2}{*}{ Type } & \multicolumn{2}{|c|}{ Sequence } & \multirow[b]{2}{*}{$n$} & \multirow[b]{2}{*}{$\boldsymbol{R}$} & \multirow[b]{2}{*}{ Type } & \multicolumn{2}{|c|}{ Sequence } \\
\hline & & & $n=$ & $R \neq$ & & & & $n=$ & $R \neq$ & & & & $\begin{array}{l}n \\
=\end{array}$ & $R \neq$ \\
\hline \multirow[t]{2}{*}{2} & 3 & $p$ & & & \multirow[t]{2}{*}{35} & 3 & $p$ & & & \multirow[t]{2}{*}{71} & 3 & $c$ & $2+69 j$ & 3 \\
\hline & 9 & $p$ & & & & 9 & $p$ & & & & 9 & $p$ & & \\
\hline \multirow[t]{2}{*}{5} & 3 & $p$ & & & \multirow[t]{2}{*}{38} & 3 & $p$ & & & \multirow[t]{2}{*}{74} & 3 & $p$ & & \\
\hline & 9 & $p$ & & & & 9 & $p$ & & & & 9 & $c$ & $11+21 j$ & 9 \\
\hline \multirow[t]{2}{*}{8} & 3 & $p$ & & & \multirow[t]{2}{*}{41} & 3 & $c$ & $20+21 j$ & 3 & \multirow[t]{2}{*}{77} & 3 & $p$ & & \\
\hline & 9 & $p$ & & & & 9 & $p$ & & & & 9 & $c$ & $20+57 j$ & 9 \\
\hline \multirow[t]{2}{*}{11} & 3 & $p$ & & & \multirow[t]{2}{*}{44} & 3 & $p$ & & & \multirow[t]{2}{*}{80} & 3 & $c$ & $14+33 j$ & 3 \\
\hline & 9 & $c$ & $11+21 j$ & 9 & & 9 & $p$ & & & & 9 & $p$ & & \\
\hline \multirow[t]{2}{*}{14} & 3 & $c$ & $14+33 j$ & 3 & \multirow[t]{2}{*}{47} & 3 & $c$ & $14+33 j$ & 3 & \multirow[t]{2}{*}{83} & 3 & $c$ & $20+21 j$ & 3 \\
\hline & 9 & $p$ & & & & 9 & $p$ & & & & 9 & $p$ & & \\
\hline \multirow[t]{2}{*}{17} & 3 & $p$ & & & \multirow[t]{2}{*}{50} & 3 & $p$ & & & 86 & 3 & $p$ & & \\
\hline & 9 & $p$ & & & & 9 & $p$ & & & & 9 & $c$ & $20+33 j$ & 9 \\
\hline 20 & 3 & $c$ & $20+21 j$ & 3 & 53 & 3 & $c$ & $14+39 j$ & 3 & 89 & 3 & $c$ & $32+57 j$ & 3 \\
\hline & 9 & $c$ & $20+33 j$ & 9 & & 9 & $c$ & $\begin{array}{l}11+21 j \\
20+33 j\end{array}$ & 9 & & 9 & $c$ & $89+93 j$ & 9 \\
\hline 23 & 3 & $p$ & & & 56 & 3 & $p$ & & & 92 & 3 & $c$ & $14+39 j$ & 3 \\
\hline & 9 & $p$ & & & & 9 & $p$ & & & & 9 & $p$ & & \\
\hline 26 & 3 & $p$ & & & 59 & 3 & $p$ & & & 95 & 3 & $p$ & & \\
\hline & 9 & $p$ & & & & 9 & $p$ & & & & 9 & $c$ & $11+21 j$ & 9 \\
\hline 29 & 3 & $p$ & & & 62 & 3 & $c$ & $20+21 j$ & 3 & 98 & 3 & $p$ & & \\
\hline & 9 & $c$ & $29+39 j$ & 9 & & 9 & $c$ & $11+51 j$ & 9 & & 9 & $c$ & $29+69 j$ & 9 \\
\hline 32 & 3 & $c$ & $32+51 j$ & 3 & 65 & 3 & $p$ & & & 101 & 3 & $p$ & & \\
\hline & 9 & $c$ & $11+21 j$ & 9 & & 9 & $p$ & & & & 9 & $p$ & & \\
\hline & & & & & 68 & 3 & $p$ & & & & & & & \\
\hline & & & ime & & & 9 & $c$ & $29+39 j$ & 9 & & & & osite & \\
\hline
\end{tabular}

Table 3(b). $n=3 t+2$ 


\begin{tabular}{|c|c|c|c|}
\hline$p$ & $n$ & $\boldsymbol{R}$ & Remarks \\
\hline \multirow{2}{*}{$\begin{array}{l}6719980305 \\
5971396836 \\
1666935769\end{array}$} & $3 t+1$ & \multirow[b]{2}{*}{9} & \multirow{2}{*}{$\begin{array}{l}\text { This } n \text { could yield additional primes with } R=1,3 \text {, or } 7 . \\
\qquad j=3199990621712923658865080646\end{array}$} \\
\hline & $10+21 j$ & & \\
\hline \multirow{2}{*}{$\begin{array}{l}5214196228 \\
5665768942 \\
3872613771\end{array}$} & $3 t+1$ & \multirow{2}{*}{1} & \multirow{2}{*}{$\begin{array}{l}\text { This } n \text { could yield additional primes with } R=7 \text { or } 9 \text {, but } \\
R=3 \text { can never be prime with this } n \text {. } \\
\qquad j=24829505850317417568685107684\end{array}$} \\
\hline & $13+21 j$ & & \\
\hline \multirow{2}{*}{$\begin{array}{l}1157569866 \\
6830365787 \\
8962467957\end{array}$} & $3 t$ & \multirow{2}{*}{7} & \multirow[t]{2}{*}{$\begin{array}{l}\text { Main } m \text { sequence prevents } R=3 \text { or } 9 \text { from forming } \\
\text { primes with this } n \text {; the imbedded sequence has } R \neq 1 \text { so } \\
\text { that } R=7 \text { is the only } R \text { which forms a prime with this } n \text {. }\end{array}$} \\
\hline & $9+21 j$ & & \\
\hline \multirow{2}{*}{$\begin{array}{l}5908726128 \\
2517955133 \\
6102196593\end{array}$} & $3 t+2$ & \multirow{2}{*}{3} & \multirow[t]{2}{*}{$\begin{array}{l}\text { Main } m \text { sequence prevents } R=1 \text { or } 7 \text { from forming } \\
\text { primes with this } n \text {; the imbedded sequence has } R \neq 9 \text { so } \\
\text { that } R=3 \text { is the only } R \text { which forms a prime with this } n \text {. }\end{array}$} \\
\hline & $11+21 j$ & & \\
\hline
\end{tabular}

Table 4. Some 30-digit primes

\begin{tabular}{|c|c|c|c|}
\hline$p$ & $n$ & $\boldsymbol{R}$ & Remarks \\
\hline $\begin{array}{l}6449532778 \\
1887693539 \\
7385586910\end{array}$ & $3 t+2$ & \multirow{2}{*}{9} & \multirow{2}{*}{$\begin{array}{l}\text { Main sequence prevents primes with } R=1 \text { or } 7 \text {. The } \\
\text { imbedded sequence give a high yield for } R=3 \text { or } 9 \\
\text { which permits this } n \text { to form a prime with } R=3 \text {. } \\
j=307120608247084254951135993766984948111374130002\end{array}$} \\
\hline $\begin{array}{l}6683910338 \\
8567300449\end{array}$ & $2+21 j$ & & \\
\hline \multirow{2}{*}{$\begin{array}{l}3076254225 \\
0301270692 \\
0514605395 \\
8616692729 \\
1732754961\end{array}$} & $3 t$ & \multirow[t]{2}{*}{1} & \multirow{2}{*}{$\begin{array}{l}\text { Main sequence prevents primes with } R=3 \text { or } 9 \text {. The } \\
\text { imbedded sequence permits this to form primes with } \\
R=1 \text { or } 7 \text {. } \\
j=93219825000912941491065031938139899779671917439\end{array}$} \\
\hline & $9+33 j$ & & \\
\hline \multirow{2}{*}{$\begin{array}{l}1545241701 \\
1775787851 \\
9510473095 \\
6315938884 \\
0946309807\end{array}$} & $3 t+1$ & \multirow[b]{2}{*}{7} & \multirow[t]{2}{*}{$\begin{array}{l}\text { The two sequences allow this to have } R=3,7 \text { or } 9 \text { to } \\
\text { form primes with this } n \text {. }\end{array}$} \\
\hline & $28+33 j$ & & \\
\hline
\end{tabular}

Table 5. Some 50-digit primes 


\section{Concluding comments}

It is clear that the various sequences occur because of the integer structure [2]. If we compare the modular rings, then the row sequences are sustained to infinity, and so the imbedded sequences should survive in the same way. This type of structural analysis is independent of the size of a domain.

\section{References}

[1] Leyendekkers, J. V. \& Shannon, A.G. (2008) Analysis of Primes Using Right-End-Digits and Integer Structure. Notes on Number Theory \& Discrete Mathematics, 14 (3), 1-10.

[2] Shannon, A. G., \& Leyendekkers, J. V. (2018) The Fibonacci Numbers and Integer Structure. New York: Nova Science Publishers, Chs. 2, 3, 6. 